\title{
GSTP1 negatively regulates Stat3 activation in epidermal growth factor signaling
}

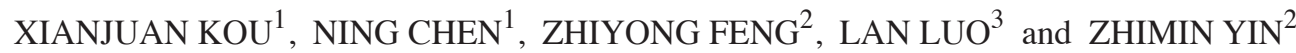 \\ ${ }^{1}$ College of Health Science, Wuhan Institute of Physical Education, Wuhan 430079; ${ }^{2}$ Jiangsu Province \\ Key Laboratory for Molecular and Medicine Biotechnology, College of Life Sciences, Nanjing \\ Normal University, Nanjing, Jiangsu 210046; ${ }^{3}$ State Key Laboratory of Pharmaceutical Biotechnology, \\ School of Life Sciences, Nanjing University, Nanjing, Jiangsu 210093, P.R. China
}

Received October 5, 2012; Accepted December 21, 2012

DOI: $10.3892 / \mathrm{ol} .2012 .1098$

\begin{abstract}
Glutathione S-transferases (GSTs) are the enzymes that defend cells against the damage mediated by oxidant and electrophilic carcinogens. GST $\pi$ (GSTP1) is a member of the GST family and the hypermethylation GSTP1 CpG island DNA is detected in human hepatocellular carcinoma (HCC) tissues, which contributes to the negative expression of GSTP1 mRNA and protein. GSTP1 expression is considered to be an early event in HCC. Stat3, a member of the signal transduction and activator of transcription (Stat) family, is important for promoting the proliferation, survival and other biological processes of cells triggered by cytokines and growth factors. Activated Stat 3 may participate in oncogenesis. Previous studies have demonstrated that overexpression of phosphorylated Stat 3 is important in the proliferation of HCC cells, suggesting that disturbance of the Stat 3 pathway may be an early event. We hypothesize that the suppression of GSTP1 expression in HCC cells increases Stat 3 activation. In order to test this hypothesis, HepG2 cells were genetically modified to transiently express high levels of GSTP1. The transient expression of GSTP1 specifically downregulated epidermal growth factor (EGF)-mediated tyrosine phosphorylation of Stat3, and subsequently suppressed the transcriptional activity of Stat3. By contrast, GSTP1 RNAi was able to lead to an increase in the phosphorylation of Stat3. In addition, overexpression of GSTP1 was capable of reducing the survival of HepG2 cells and inducing cell cycle arrest. This inhibition was mediated
\end{abstract}

Correspondence to: Professor Zhimin Yin, College of Life Sciences, Nanjing Normal University, 1 Wenyuan Road, Nanjing 210046, P.R. China

E-mail: yinzhimin@njnu.edu.cn

Professor Lan Luo, State Key Laboratory of Pharmaceutical Biotechnology, School of Life Sciences, Nanjing University, 22 Hankou Road, Nanjing 210093, P.R. China

E-mail: lanluo@nju.edu.cn

Key words: glutathione S-transferase $\pi$, Stat 3 , phosphorylation, cell cycle by a direct interaction between GSTP1 and Stat3. Overall, our results suggest that GSTP1 is important in the regulation of the transcriptional activity of Stat3, and that it is also a regulator of the cell cycle via EGF signaling.

\section{Introduction}

Glutathione S-transferases (GSTs), a superfamily of detoxifying enzymes, contain at least five subclasses, including $\alpha$, $\mu, \pi, \omega$ and $\theta$. GSTs act catalytically through the nucleophilic attachment of the sulfur atom of glutathione (GSH) onto the electrophilic groups of substrate molecules $(1,2)$. GSTs are important in protecting cells from cytotoxic and carcinogenic agents, removing oxidative stress products, and modulating cell proliferation and signaling pathways $(2,3)$. As an isozyme of GST, GSTP1 is a major regulator of cell signaling in response to stress, hypoxia, growth factors and other stimuli. Previous studies have demonstrated that GSTP1 inhibits lipopolysaccharide-induced MAPK, and that NF- $\kappa \mathrm{B}$ activation decreases LPS-induced iNOS production by regulating MAPK activation (4). In addition, GSTP1 expression is highly correlated with carcinogenesis; GSTP1 is overexpressed in a variety of human cancers, including lung, colon, ovary, bladder and kidney cancer (5-8). By contrast, the reduced expression and activity of GSTP1 are observed due to the hypermethylation of its promoter in hepatocellular carcinoma (HCC) and prostate cancer (9-10), although GSTP1 may also be detected in the corresponding non-tumorous tissues. However, GSTP1 null mice reveal an increased risk of carcinogen-induced skin tumorigenesis (11). Notably, the overexpression of GSTP1 has been reported to protect prostate cells from cytotoxicity and DNA damage due the heterocyclic amine carcinogen PhIP (12), which suggests that silencing of the GSTP1 gene by $\mathrm{CpG}$ island DNA methylation may be important in the development of HCC.

The signal transducer and activator of transcription (Stat) family of cytoplasmic proteins is important for promoting the proliferation, survival, and other biological processes triggered by cytokines and growth factors, including epidermal growth factor (EGF) (13-15). EGF induces the activation of Stat1, Stat3 and Stat5 in cancer cells. Stat 3 has been demonstrated to play a critical role in EGF signaling in 
both normal and tumor cells (16). Normal Stat activation is a highly regulated process. However, atypical activation of Stat 3 is usually detected in various human tumors including HCC, and may modulate the oncogenic transformation and progression (17). Furthermore, Stat 3 has been implicated as a promising target for HCC therapy, as the inhibition of Stat 3 has been shown to induce growth arrest and apoptosis of human HCC cells (18). Since GSTP1 exerts important anti-inflammatory, antioxidant and detoxification functions in the body, and its promoter is hypermethylated in $\mathrm{HCC}$, the restoration of GSTP1 expression may be a promising method for preventing tumors. In the present study, the possible regulatory mechanisms of GSTP1 on Stat activation have been explored in HepG2 cells. The results indicate that the overexpression of GSTP1 specifically downregulates Stat3 activation, and inhibits cell growth via a direct interaction between GSTP1 and Stat3.

\section{Materials and methods}

Antibodies and reagents. The p-Stat 3 (Y705), Stat 3 and cyclin D1 antibodies were purchased from Cell Signaling Technology, Inc. (Beverly, MA, USA). A mouse monoclonal antibody against xpress-tag was purchased from Invitrogen Life Technologies (Carlsbad, CA, USA). Flag-tag was purchased from Sigma (St. Louis, MO, USA). GAPDH and protein $\mathrm{G}$ were purchased from Roche Applied Science (Indianapolis, IN, USA). Mouse or rabbit A/G and $\mathrm{IgG}$ antibodies were purchased from Santa Cruz Biotechnology, Inc. (Santa Cruz, CA, USA). Secondary antibodies coupled to IRDye 800 fluorophore for the Odyssey Infrared Imaging System were purchased from Rockland Immunochemicals, Inc. (Gilbertsville, PA, USA).

Plasmid construction. Flag-Stat3 (wt) was provided by Dr Zhijie Chang of Tsinghua University (Beijing, China). GSTP1-RNAi was constructed into pRNA-u6. All expression plasmids were confirmed by sequencing and purified by the Endofree Plasmid Preparation kit (Qiagen, Hilden, Germany).

Cell culture and transfection. HEK293, HepG2 and WRL-68 cell lines were purchased from the Institute of Biochemistry and Cell Biology, the Chinese Academy of Sciences (Shanghai, China), and then cultured in Dulbecco's Modified Eagle's Medium (DMEM; Invitrogen Life Technologies) supplemented with $10 \%$ fetal bovine serum (FBS; HyClone Laboratories; Logan, UT, USA), $100 \mathrm{U} / \mathrm{ml}$ penicillin and $100 \mu \mathrm{g} / \mathrm{ml}$ streptomycin in $5 \% \mathrm{CO}_{2}$ at $37^{\circ} \mathrm{C}$. Transient transfection was performed using the Lipofectamine 2000 reagent (Invitrogen Life Technologies) according to the manufacturer's instructions. In all cases, the total amount of DNA was normalized by the empty control plasmids.

Immunoprecipitation and immunoblotting analysis. HEK293 cells were washed twice with ice-cold phosphate-buffered saline (PBS; pH 7.4) and lysed in lysis buffer containing $20 \mathrm{mM}$ Tris ( $\mathrm{pH}$ 7.5), $135 \mathrm{mM} \mathrm{NaCl}, 2 \mathrm{mM}$ EDTA, $2 \mathrm{mM}$ dithiothreitol (DTT), $25 \mathrm{mM} \beta$-glycerophosphate, $2 \mathrm{mM}$ sodium pyrophosphate, $10 \%$ glycerol, $1 \%$ Triton X-100, $1 \mathrm{mM}$ sodium orthovanadate, $10 \mathrm{mM} \mathrm{NaF}$ and $1 \mathrm{mM}$ phenylmeth- ylsulfonyl fluoride (PMSF), supplemented with complete protease inhibitor cocktail (Roche Applied Science). Following incubation on ice for $30 \mathrm{~min}$, the cell lysates were centrifuged at $15,000 \mathrm{x} \mathrm{g}$ at $4^{\circ} \mathrm{C}$ for $15 \mathrm{~min}$. Proteins $(500 \mu \mathrm{g})$ were immunoprecipitated with the designated antibodies, respectively. The precleared Protein A/G PLUS-Agarose beads (Santa Cruz Biotechnology, Inc.) were incubated with immunocomplexes for $2 \mathrm{~h}$ and washed four times with the lysis buffer. The immunoprecipitates were subjected to sodium dodecyl sulfate-polyacrylamide gel electrophoresis (SDS-PAGE), then transferred onto a nitrocellulose membrane (Hybond-C; Amersham Biosciences Corp.; Piscataway, NJ, USA). The immunoblotting analyses were performed. The results were visualized using IRDye 800 fluorophore-conjugated antibody in the Li-COR Odyssey Infrared Imaging System according to the manufacturer's instructions (LI-COR Biosciences; Lincoln, NE, USA).

Cell cycle assay. Cells were collected by trypsinization, pelleted at $800 \mathrm{x} \mathrm{g}$ for $10 \mathrm{~min}$ and fixed in $70 \%$ ethanol. The DNA content was evaluated by flow cytometry with propidium iodide (PI) staining. Flow cytometric analysis was performed using FACScan (Becton-Dickinson; Mountain View, CA, USA) with Cell Quest software.

Cell viability. The transfected HepG2 cells were seeded in 96-well plates and the cell viability was evaluated by a 3-(4,5-dimethylthiazol-2-yl)-2,5-diphenyltetrazolium bromide (MTT) assay. For each experiment, six wells were used and the experiments were repeated three times.

Statistical analysis. All experimental data was obtained from cultured cells were expressed as mean \pm SD. Western blotting analysis experiments were repeated 3 times with similar trends. A one-way repeated measure analysis ofvariance and a Student's t-test were used to determine the significance of the difference between two groups.

\section{Results}

Overexpression of GSTP1 inhibits EGF-induced Stat3 activation. In order to explore the effect of GSTP1 on endogenous Stat 3 activation in HepG2 cells, the cells were transfected with xpress-tagged GSTP1 followed by EGF stimulation. The phosphorylation of Stat3 was examined by western blot analysis. Overexpression of GSTP1 inhibited the EGF-stimulated tyrosine phosphorylation of Stat 3 in a dose-dependent manner (Fig. 1A). However, serine phosphorylation of Stat3 and a change in the expression of Stat3 were not observed. In order to explore whether GSTP1 is able to modulate Stat3 transcriptional activity in the presence of EGF, HepG2 cells were co-transfected with Stat3-dependent luciferase reporter gene and an xpress-GSTP1 plasmid. As is demonstrated in Fig. 1B, the cells were stimulated by EGF for $15 \mathrm{~min}$ and a 3-fold enhancement in fluorescence intensity was observed when compared with the control cells. However, the increase of fluorescence intensity was blocked in the presence of exogenous GSTP1. These results indicate that the suppression of Stat 3 transcription may result from the inhibition of its tyrosine phosphorylation. 

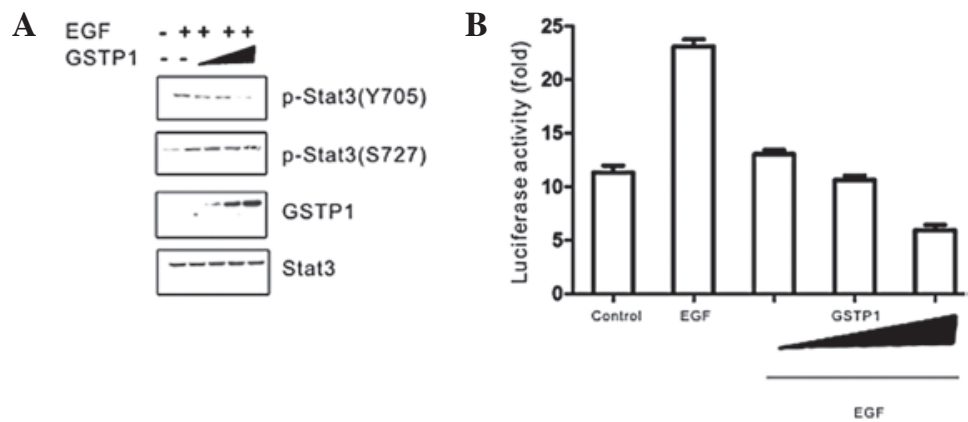

Figure 1. Overexpression of glutathione S-transferase $\pi$ (GSTP1) suppresses epidermal growth factor (EGF)-induced Stat3 activity. (A) HepG2 cells were transfected with the xpress-tagged GSTP1 expression plasmid $(0.5,1$ and $2 \mu \mathrm{g})$. The cells were either left untreated or treated with EGF (100 ng/ml) for $15 \mathrm{~min}$. Total cell lysates were prepared and subjected to western blot analysis using antibody against phospho-Tyr-705-Stat3 (p-Stat3 Y705) or phospho-Ser-727-Stat3 (p-Stat3S727). (B) GSTP1 suppresses EGF-induced Stat3 promoter activity. The HepG2 cells in 12-well plates were transiently transfected with different quantities of the xpress-tagged GSTP1 expression plasmid together with the pCMV- $\beta$-gal control vector and Stat 3 reporter plasmid. Twenty-four hours following transfection, the cells were stimulated for $15 \mathrm{~min}$. Cell lysates were prepared to measure luciferase activity using the Luciferase Assay System (Promega Corporation; Madison, WI, USA) and analyzed by the Luminometer TD-20/20 (Turner Biosystems, Inc.; Sunnyvale, CA, USA). Luciferase activity was normalized to $\beta$-gal activity.

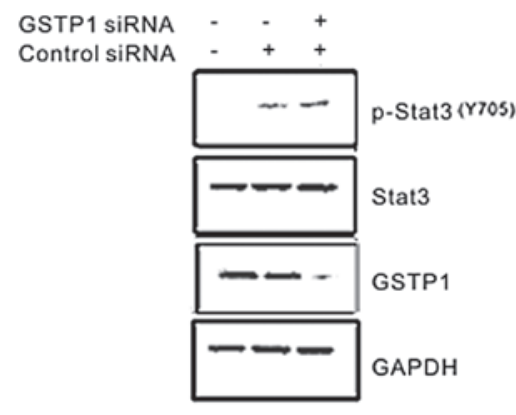

Figure 2. Knockdown of endogenous glutathione S-transferase $\pi$ (GSTP1) expression enhances tyrosine phosphorylation of Stat 3 stimulated by epidermal growth factor (EGF). WRL-68 cells were transfected with $40 \mu 1$ of $20 \mu \mathrm{M}$ siRNA of GSTP1 or the control siRNA duplex with Lipofectamine 2000 reagent for $12 \mathrm{~h}$. Cells were transferred to a normal culture medium for $14 \mathrm{~h}$ and treated with EGF for $30 \mathrm{~min}$ before harvesting.

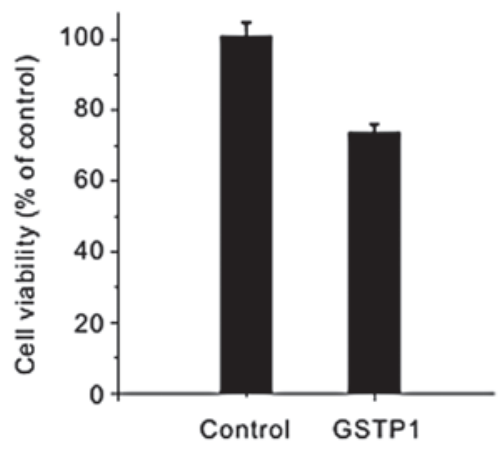

Figure 3. Glutathione S-transferase $\pi$ (GSTP1) overexpression results in a reduction of proliferation and an increase in the number of cells in the G0/G1 phase. The HepG2 cells were transfected with empty vector and wild-type GSTP1 prior to treatment with epidermal growth factor (EGF). The extent of cell viability was assessed by the 3-(4,5-dimethylthiazol-2-yl)2,5-diphenyltetrazolium bromide (MTT) assay. In each experiment, averages of eight replicates were normalized to the average of the vector control. Data are presented as mean \pm standard error of the mean from $\geq 3$ independent experiments. $\mathrm{P}<0.05$ compared with the vector control.

GSTP1 knockdown increases tyrosine phosphorylation of Stat3 stimulated by EGF. In order to further confirm whether GSTP1 downregulated the phosphorylation of Stat3, the
A

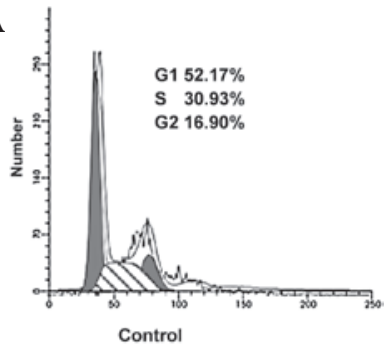

B

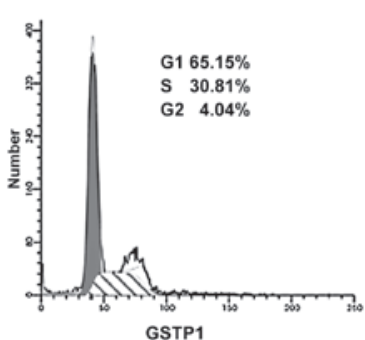

Figure 4. Glutathione S-transferase $\pi$ (GSTP1) overexpression results in cell cycle arrest. The HepG2 cells were transfected with an empty vector and wild-type GSTP1 $(1 \mu \mathrm{g})$, collected $24 \mathrm{~h}$ after transfection, stained with propidium iodide (PI) and then analyzed by flow cytometry. The percentage of cells in each phase of the cell cycle is shown.

GSTP1 siRNA was transfected into WRL-68 cells that have higher endogenous levels of Stat3. The effect of GSTP1 siRNA on EGF-mediated tyrosine phosphorylation of Stat3 was examined. As expected, the expression of GSTP1 was effectively blocked by GSTP1 siRNA, and GSTP1 siRNA further enhanced the EGF-stimulated tyrosine phosphorylation of Stat3 (Fig. 2). By contrast, GSTP1 siRNA had no effect on the expression level of Stat3. These results indicated that endogenous GSTP1 negatively regulated EGF-induced Stat3 activation.

Effects of forced expression of GSTP1 on the cell proliferation and cell cycle phase distribution in HepG2 cells. Since GSTP1 inhibits Stat3-dependent luciferase activity and previous studies have demonstrated that GSTP1 inhibits cell proliferation (19), the effect of GSTP1 on cell viability was also examined. The HepG2 cells were transfected with $2 \mu \mathrm{g}$ 


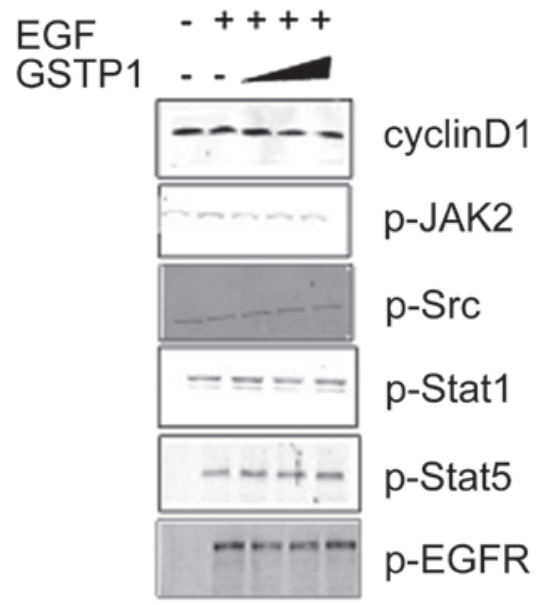

Figure 5. Effect of glutathione S-transferase $\pi$ (GSTP1) overexpression on upstream kinases and cyclin D1. The cell lysates from HepG2 cells treated with GSTP1 for $48 \mathrm{~h}$ were resolved on $10 \%$ sodium dodecyl sulfate-polyacrylamide gel electrophoresis (SDS-PAGE), then immunoblotted with antibodies as indicated in Materials and methods. EGF, epidermal growth factor; p, phosphorylated: EGFR, EGF receptor.
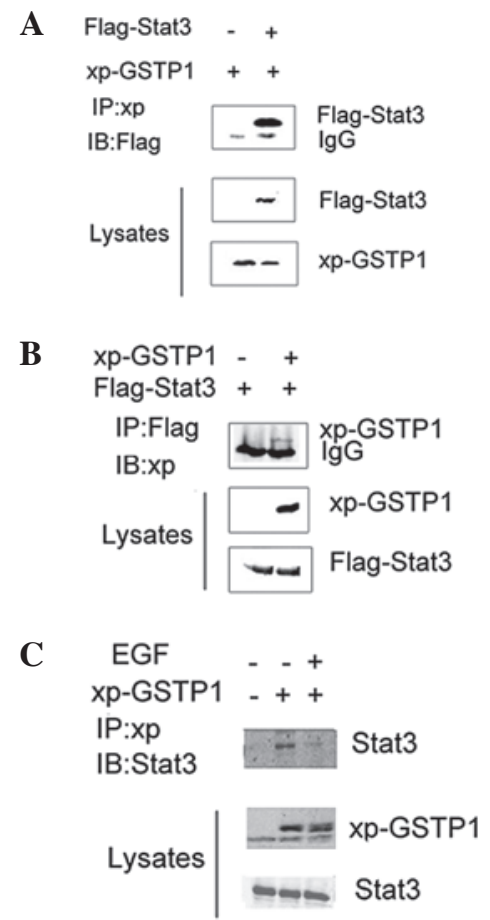

Figure 6. Glutathione S-transferase $\pi$ (GSTP1) physically associates with Stat3. HEK293 cells were co-transfected with Flag-Stat3 (A) and xpress (xp)-GSTP1 (B) or empty expression vectors as indicated. Thirty-six hours following transfection, cell lysates were incubated with protein $A / G$ PLUS-agarose beads conjugated with anti-xp antibody (A) and with protein A/G-Agarose beads conjugated with anti-FLAG antibody (B). The immunoprecipitated proteins were immunoblotted with anti-FLAG (A) or anti-xp (B) antibody. (C) xp-GSTP1 (1 $\mu \mathrm{g})$-transfected HepG2 cell lysates were subjected to immunoprecipitation with anti-xp, and then the precipitates were analyzed by immunoblotting with anti-xp antibody. The cell lysates were immunoblotted with anti-Stat 3 antibody or anti-xp antibody. IB, immunoblotting; IP, immunoprecipitation; IgG, immunoglobulin G. Similar results were observed in three independent experiments. EGF, epidermal growth factor.

GSTP1 for $36 \mathrm{~h}$ and the GSTP1-transfected cells exhibited a reduced proliferation compared with the control HepG2 cells (Fig. 3), which suggested that GSTP1 possessed the function of suppressing cell proliferation. Additionally, Stat3 is important in cell cycle progression, whereas the inhibition of constitutively-active Stat 3 induces cell cycle arrest $(20,21)$. Flow cytometric analyses (Fig. 4) revealed that overexpression of GSTP1 induces cell cycle arrest and cell accumulation at the G0-G1 phase by $36 \mathrm{~h}$ when compared with untransfected cells. These results suggest that the GSTP1-induced inhibition of Stat3 signaling may result in the inhibition of cell growth and blockage of the cell cycle.

GSTP1 expression inhibits Stat3 but not its upstream regulators. It is well known that the phosphorylation of Stats depends on the activation of JAKs and/or Src family kinases, which stimulated us to explore the possible tyrosine kinases involved in the activation of Stats through GSTP1 inhibition. In order to gain insights into the inhibitory mechanism of GSTP1 on the Stat signaling cascade, the effect of GSTP1 on JAK and Src kinase activity was also examined. As demonstrated in Fig. 5, GSTP1 suppressed Stat3-mediated downstream factor cyclin D1, and did not affect the upstream regulators, such as p-JAK2, p-Src and p-EGFR, of the phosphorylation of Stat3. In addition, the phosphorylation of Stat5 was not affected by GSTP1, which revealed the specificity of GSTP1 for the phosphorylation of Stat3. Therefore, GSTP1 may inhibit the phosphorylation of Stat 3 by direct inhibition at its protein level.

Inhibition of Stat 3 activity involves a direct interaction between GSTP1 and Stat3. In order to understand the mechanism of suppression of Stat 3 activation, HEK293 cells transiently co-transfected with Flag-Stat 3 and Xpress-GSTP1 were subjected to immunoprecipitation with anti-Flag or -Xpress antibody to explore the association between GSTP1 and Stat3. The immunoprecipitates were separated by SDS-PAGE, and transferred to a nitrocellulose membrane. The results indicated that GSTP1 co-immunoprecipitated with Stat3 in HEK293 cells (Figs. 6A and B). Similarly, a specific association between GSTP1 and endogenous Stat3 was observed in Xpress-GSTP1-transfected HepG2 cells. The co-immunoprecipitation assay demonstrated that XpressGSTP1 was able to physically interact with endogenous Stat3 (Fig. 6C). These results suggest that the negative regulatory effect of GSTP1 on Stat3 is mediated by a physical interaction between the two proteins.

\section{Discussion}

GSTs are a superfamily of detoxifying enzymes that catalyze the conjugation of reduced GSH via a variety of electrophiles. In addition to their catalytic functions, GSTs also serve as nonenzymatic binding proteins, interacting with various lipophilic compounds including steroid and thyroid hormones (22-25). Furthermore, GSTP1 also regulates important normal cellular functions through its interaction with a number of critical cellular proteins, such as transglutaminase 2 (TGM2), apoptosis signal-regulating kinase 1 (ASK1) and Fanconi anemia group C protein (FANCC) (25). These findings suggest that the diverse functions of GSTP1 may be determined by the interactions with its key partner proteins. The currently 
identified mechanisms of Stat3 inhibition include dephosphorylation, inactivation of JAK by suppressor of cytokine signaling (SOCS1) protein (26) and abrogation of DNA binding by the protein inhibitor of activated Stat (PIAS) (27). However, in the present study, the physical interaction between GSTP1 and Stat 3 resulted in the suppression of Stat 3 activity (Fig. 6). Notably, upon treatment of HepG2 cells with EGF, a disassociation of the GSTP1/Stat 3 complex was achieved, suggesting that Stat 3 forms a complex with GSTP1 and that EGF may release Stat 3 from the GSTP1 binding complex. The mechanism whereby GSTP1 specifically interacts with Stat 3 may present a novel means of therapeutic intervention in Stat3-driven tumors.

HCC is the most common type of primary liver cancer, particularly in developing countries. More than half of cancer patients are identified as having HCC in China (28). Previous studies have reported that Stat 3 is a promising target for $\mathrm{HCC}$ therapy (18). GSTP1 is downregulated or absent in HCC due to the action of DNA methyltransferase. However, the clinical applications of nucleoside analogs used as DNA methyltransferase inhibitors are limited somewhat by myelosuppression and other potential side effects. In the present study, the restoration of the GSTP1 protein has been demonstrated to exert an anticancer effect by inhibiting the Stat 3 signaling pathway in HCC cells. We have demonstrated that the overexpression of GSTP1 in HepG2 cells suppresses the tyrosine phosphorylation and transcription activity of EGF-inducible Stat3, as well as the gene expression of Stat3-regulated cyclin D1, thus resulting in the inhibition of proliferation and increased accumulation of cells in the G1/G0 phase (Fig. 4). JAK2 or Src may be common upstream effectors for the activation of Stat3. Our results demonstrated that GSTP1 only inhibited Stat3, but no JAK2-dependent mechanism was observed in the transfected cells (Fig. 5).

In summary, a novel function of GSTP1 in inhibiting EGF-induced Stat 3 activation has been demonstrated. The GSTP1-Stat 3 complex reduces proliferation and arrests the cell cycle by terminating Stat 3 activity.

\section{Acknowledgements}

The authors would like to thank Dr Zhijie Chang (Tsinghua University) for providing the plasmid constructs used in this study. This study was supported by grants from the National Nature Science Foundation of China (Nos. 30770842 and 30771979).

\section{References}

1. Tew KD: Glutathione-associated enzymes in anticancer drug resistance. Cancer Res 54: 4313-4320, 1994.

2. Townsend D and Tew K: Cancer drugs, genetic variation and the glutathione-S-transferase gene family. Am J Pharmacogenomics 3: 157-172, 2003.

3. Eaton DL and Bammler TK: Concise review of the glutathione $\mathrm{S}$-transferases and their significance to toxicology. Toxicol Sci 49: 156-164, 1999.

4. Xue B, Wu Y, Yin Z, Zhang H, Sun S, Yi T and Luo L: Regulation of lipopolysaccharide-induced inflammatory response by glutathione S-transferase P1 in RAW264.7 cells. FEBS Lett 579: 4081-4087, 2005.
5. Inoue $\mathrm{T}$, Ishida $\mathrm{T}$, Suqio $\mathrm{K}$, Maehara $\mathrm{Y}$ and Suqimachi $\mathrm{K}$ : Glutathione $\mathrm{S}$ transferase $\mathrm{Pi}$ is a powerful indicator in chemotherapy of human lung squamous-cell carcinoma. Respiration 62: 223-227, 1995.

6. Sato K: Glutathione transferases as markers of preneoplasia and neoplasia. Adv Cancer Res 52: 205-255, 1989.

7. Singh SV, Xu BH, Gupta V, Emerson EO, Zaren H and Jani JP: Characterization of a human bladder cancer cell line selected for resistance to BMY 25067, a novel analogue of mitomycin C. Cancer Lett 95: 49-56, 1995.

8. Grignon DJ, Abdel-Malak M, Mertens WC, Sakr WA and Shepherd RR: Glutathione S-transferase expression in renal cell carcinoma: a new marker of differentiation. Mod Pathol 7: 186-189, 1994.

9. Zhong S, Tang MW, Yeo W, Liu C, Lo YM and Johnson PJ: Silencing of GSTP1 gene by CpG island DNA hypermethylation in HBV-associated hepatocellular carcinomas. Clin Cancer Res 8: 1087-1092, 2002.

10. Lin X, Asgari K, Putzi MJ, et al: Reversal of GSTP1 CpG island hypermethylation and reactivation of pi-class glutathione S-transferase (GSTP1) expression in human prostate cancer cells by treatment with procainamide. Cancer Res 61: 8611-8616, 2001.

11. Henderson CJ, Smith AG, Ure J, Brown K, Bacon EJ and Wolf CR: Increased skin tumorigenesis in mice lacking pi class glutathione S-transferases. Proc Natl Acad Sci USA 95: 5275-5280, 1998.

12. Nelson CP, Kidd LC, Sauvageot J, et al: Protection against 2-hydroxyamino-1-methyl-6-phenylimidazo[4,5-b]pyridine cytotoxicity and DNA adduct formation in human prostate by glutathione S-transferase P1. Cancer Res 61: 103-109, 2001.

13. Bromberg J and Darnell JE Jr: The role of STATs in transcriptional control and their impact on cellular function. Oncogene 19: 2468-2473, 2000.

14. Darnell JE Jr: Transcription factors as targets for cancer therapy. Nat Rev Cancer 2: 740-749, 2002.

15. Yu H and Jove R: The STATs of cancer - new molecular targets come of age. Nat Rev Cancer 4: 97-105, 2004.

16. Zhang T, Ma J and Cao X: Grb2 regulates Stat3 activation negatively in epidermal growth factor signalling. Biochem J 376: 457-464, 2003.

17. Buettner R, Mora LB and Jove R: Activated STAT signaling in human tumors provides novel molecular targets for therapeutic intervention. Clin Cancer Res 8: 945-954, 2002.

18. Li F, Fernandez PP, Rajendran P, Hui KM and Sethi G: Diosgenin, a steroidal saponin, inhibits STAT3 signaling pathway leading to suppression of proliferation and chemosensitization of human hepatocellular carcinoma cells. Cancer Lett 292: 197-207, 2010.

19. Ruscoe JE, Rosario LA, Wang T, et al: Pharmacologic or genetic manipulation of glutathione S-transferase P1-1 (GSTpi) influences cell proliferation pathways. J Pharmacol Exp Ther 298: 339-345, 2001.

20. Garcia R, Bowman TL, Niu G, et al: Constitutive activation of Stat 3 by the Src and JAK tyrosine kinases participates in growth regulation of human breast carcinoma cells. Oncogene 20: 2499-2513, 2001.

21. Niu G, Bowman T, Huanq M, et al: Roles of activated Src and Stat3 signaling in melanoma tumor cell growth. Oncogene 21: 7001-7010, 2002.

22. Ishigaki S, Abramovitz M and Listowsky I: GlutathioneS-transferases are major cytosolic thyroid hormone binding proteins. Arch Biochem Biophys 273: 265-272, 1989.

23. Ketley JN, Habig WH and Jakoby WB: Binding of nonsubstrate ligands to the glutathione S-transferases. J Biol Chem 250: 8670-8673, 1975 .

24. Litwack G, Ketterer B and Arias IM: Ligandin: a hepatic protein which binds steroids, bilirubin, carcinogens and a number of exogenous organic anions. Nature 234: 466-467, 1971.

25. Lo HW and Ali-Osman F: Genetic polymorphism and function of glutathione S-transferases in tumor drug resistance. Curr Opin Pharmacol 7: 367-374, 2007.

26. Naka T, Narazaki M, Hirata M, et al: Structure and function of a new STAT-induced STAT inhibitor. Nature 387: 924-929, 1997.

27. Chung CD, Liao J, Liu B, Rao X, Jav P, Berta P and Shuai K: Specific inhibition of Stat3 signal transduction by PIAS3. Science 278: 1803-1805, 1997.

28. Parkin DM, Bray F, Ferlay J and Pisani P: Global cancer statistics, 2002. CA Cancer J Clin 55: 74-108, 2005. 